\title{
ECHOES FROM TIMES PAST
}

It is planned under this heading, and as a regular feature, to include extracts from earlier issues of the Journal. The extract printed here is taken from a paper by Lt Col $R C$ Cottell RAMC (1910. Volume XIV, 549-550) entitled "A Plea for the Employment of Aurists in Connection with Recruiting".

Having the opportunity of seeing the medical documents of invalids that pass through the hands of the Chelsea Commissioners at the Royal Hospital, I have been struck by the frequency with which "deafness" has been a cause of invaliding, and also by the very short service of the invalids with this disability. I therefore thought it would be instructive to take the fresh cases of invaliding for all diseases that came before the Commissioners during the first three months of 1909 and note the comparative frequency of invaliding for "deafness" for that period.

I give a summary of my results-Between January 1, 1909, and March 31, 1909, 743 fresh cases of men invalided from the service came before the Chelsea Commissioners. Of this number sixty-two were invalids for "deafness". Two of them were long service men of sixteen and eighteen years respectivly.

The average of the remaining sixty men when invalided was 21 years, 8 months, and their average service two years and five months. The number under one year's service was ten. In eighteen cases the ear trouble was stated by the men to have been noticed "from childhood", and in addition to these eighteen cases, seventeen gave a fairly good history of the disease before enlistment.

This leaves twenty-seven cases as probably arising after enlistment. Of these twenty-seven cases, three were said to be due to bathing, two were said to follow malaria; in seventeen no cause could be suggested; four were said to be due to injury (two to "blow on the head", one to "rifle discharging" and one to "fall down a hatchway"). Of the long service men, one was said to be due to "malaria", and in the others no cause could be suggested.

In thirty-seven cases both ears were affected, in ten the right, and in fifteen the left only. There was discharge from the external meatus from both ears in twenty, from the right ear only in fourteen, and from the left only in eighteen. in twenty-two there was perforation of the tympanic membrane in both ears, in thirteen in the right only, and in seventeen in the left only. In seven deafness was very marked without either discharge or perforation. Note-Forty-eight of the men were infantrymen, twelve cavalrymen, and two garrison drummers.

Such short service as these men have given in return for their expensive training is obviously a very bad investment for the State. I think when the facts given are thoughtfully considered, my brother officers will agree that a remedy is much needed.

I am of the opinion that more care must be exercised in testing the hearing of the recruits and in examining their ears with the auroscope-which should be invariably used-and in all cases of doubt they should be seen by an aural specialist. If there are many of a doubtful character more aural specialists may have to be asked for, but, if the remedy would even halve this terrible loss, the time and money would be well spent. 